\title{
Speculative Parallel Simulation with an Adaptive Throttle Scheme
}

\author{
Seng Chuan TAY, Yong Meng TEO and Siew Theng KONG \\ Department of Information Systems \& Computer Science \\ National University of Singapore \\ Lower Kent Ridge Road \\ Singapore 119260 \\ email: taysengc@iscs.nus.sg
}

\begin{abstract}
Excessive rollback recoveries due to overoptimistic event execution in Time Warp simulators often degrade their runtime performance. This paper presents a twosided throttling scheme to dynamically adjust the event execution speed of Time Warp simulators. The proposed throttle is based on a new concept called global progress window, which allows the individual simulation process to be positioned on a global time scale, thereby to accelerate or suspend their event execution. As each simulation process can be throttled to a steady state, excessive rollback recoveries due to causality errors can be avoided. To quantify the effect of rollbacks and for purpose of comparing different Time Warp implementations, we propose two new measures called RPE (number of Rollback events Per committed Event), and $\mathcal{E}$ (relative Effectiveness in reducing rollback overhead). Our implementation results show that the proposed throttle effectively regulates the proceeding of each simulation process, resulting in a significant reduction in rollback thrashing and elapsed time.
\end{abstract}

Keywords : time warp, speculative parallelism, adaptive control scheme, parallelism throttle, global progress window

\section{Introduction}

Parallel discrete-event simulation (PDES) is performed by a set of logical processes (LP) that communicate to each other by passing timestamped messages. Each message corresponds to a simulation event or the synchronization protocol used by the simulators. Each LP has its own local clock, also called local virtual time (LVT), to indicate the progress of simulation. The LVT is advanced whenever an event is executed in the LP, and the parallel simulation is complete when all LVTs reach the duration of simulation. Two PDES mechanisms have been widely discussed: conservative [14] and optimistic [9]. The conservative approach ensures that an event execution will not cause any causality error before it is carried out. Deadlock problems may arise in conservative parallel simulations due to the blocked LPs waiting for event safety [13]. On the other hand, the optimistic approach (also called Time Warp) performs the event execution greedily without adhering to the safety constraint [4]. As the Time Warp mechanism does not enforce strict time-ordered event execution, it can potentially uncover higher degree of parallelism in the simulated system. This, in turn, may also lead to a better or even super-critical speedup which cannot be found in any conservative schemes [1T]. A repercussion of such a relaxed scheme is the excessive rollback recovery caused by the premature event execution. This problem is worst in a heterogeneous computing platform with long communication delays. In the worst case, the rollback recovery may become cascading and recursive, meaning that the Time Warp simulator may exhibit thrashing behavior. Many resolutions have been proposed to reduce rollback thrashing and its overhead. Most of the solutions adopt the one-sided approach to constrain the Time Warp optimism $[1,2,3,5,17,18]$. In addition, some form of synchronization, such as barrier or polling, is often required to bound the computations. As a result, the performance of these schemes largely depends on the events available for processing within the bounds. If the number of events available is not sufficient to outweigh the synchronization overhead, the constrained optimism may cause an undesirable performance slow down in Time Warp simulation. Dynamic load balancing technique has also been used in some Time Warp systems $[15,16]$. However, load balancing is not a feasible approach for distributed computing platforms if the migration overhead outweighs its potential gain. Other schemes decrease a LVT to an earlier time 
by undoing the processed events even without the presence of a straggler $[6,10,11]$, thus greatly wasting the CPU cycles used in the event execution. Instead of using the one-sided schemes to limit the Time Warp optimism, or using the load balancing schemes or performing the artificial rollbacks, this paper adopts a two-sided approach to accelerate the slow LPs, and suspend those processes which are run away. Our dynamic throttling scheme is based on a new concept called global progress window which allows each individual simulation process to position itself on a global time scale for adjusting its event execution speed. The main advantage of this throttling scheme is its adaptiveness to different rates of LVT progression during the simulation run. The rest of this paper is organized as follows. Section 2 reviews the existing optimism control schemes proposed in the literature. We also highlight the strengths and weaknesses of such control schemes. Section 3 discusses the proposed throttling scheme based on a new concept called global progress window, which is a global scale for each process to gauge their simulation progress. We also explain the parameterization of event execution speed, the precautions against racing effect and the throttling mechanism. Section 4 investigates the effectiveness of the proposed throttling scheme in reducing the number of rollback occurrences, We also compare the runtime performance of the throttling scheme with the conventional Time Warp scheme. Section 5 contains our concluding remarks.

\section{Adaptive Optimism Control Schemes}

Ball and Hoyt's adaptive Time Warp concurrency control scheme (ATW) restrains the rollback propagation by suspending those LPs having excessive occurrences of straggler [1]. In the ATW scheme, if the number of stragglers in a LP reaches a preset number, the LP will have to suspend its event execution for a time duration called block window. The size of a block window is adapted dynamically according to the CPU cycles lost on blocking and rollback recovery. Ferscha and Luthi use a probabilistic cost model [5] to evaluate the tradeoff between optimistically processing and conservatively blocking the simulation engine. The scheme requires each LP to monitor the rate of LVT progression, which is the change in LVT per unit CPU time, and the amount of CPU cycles used in each rollback to establish a cost model. Empirical data for rollback probability and recovery overheads are collected regularly, and used to compute the optimal duration of blocking delay. Das also develops a cost model that involves both rollback probability and the probability for an event computation performed on the critical path
[3]. The model requires the degree of optimism, which is the time distance from GVT to LVT, to be recorded in the state queue. Statistical data pertaining to committed or rolled back events can then be obtained by observing the degree of optimism each time an event is rolled back or fossil collected. By using such empirical data, the model can estimate an appropriate window size for constraining the Time Warp optimism. In general, such control schemes can adapt to the dynamism of the PDES on the fly, but nothing has been done to speed up the slow LPs. As a result, the adaptiveness mainly focuses on constraining the optimism of Time Warp simulation.

Rehier and Jefferson [15] uses effective utilization, which is the fraction of work that will not be rolled back on a given node, to balance the workload in Time Warp Operating System (TWOS). As nodes with a higher effective workload have a larger consumption of CPU power, TWOS will shift load off nodes doing more effective work onto nodes doing less effective work. Schlagenhaft, Ruhwandl, Sporrer and Bauer [16] uses the virtual time progress (VTP) to monitor the simulation process used for circuit simulation. The simulated circuit is divided into clusters, and more than one cluster is placed onto each simulation process. If the VTP indicator of a cluster exceeds a break even point, the cluster is moved from the overloaded simulation process to the underloaded one. Although such dynamic load balancing schemes are able to ensure even progress of simulation processes, they may incur tremendous migration overheads especially on distributed computing platforms.

Other adaptive schemes reclaim the allocated memory by performing a resynchronization or returning the unprocessed messages to their senders. Although their main objective is to prevent program clashes due to insufficient memory, these schemes also indirectly constrain the optimism in Time Warp. Madisetti and Hardaker propose a probabilistic scheme to resynchronize all the LPs regularly during a simulation run [12]. They use a Genie process to broadcast a timestamped SYNC message. On the receipt of the SYNC message, each $L P$ will discard all positive and negative messages with a send time greater than the timestamp, and possibly roll back without side effects. The LPs then resume event processing from the common time. Jefferson proposes a message sendback scheme [9] to return part or all of the unprocessed event messages to their senders to reclaim the memory storage used for storing newly arrived event messages. In the sendback scheme the event message with the highest timestamp is returned first as it is most likely to contain incorrect information. Gafni's protocols is an enhancement to message 
sendback where an anti-message can also be sent to annihilate its positive copy whenever memory storage is insufficient [6]. The optimism in a fast LP can therefore be constrained. A cancelback scheme, also proposed by Jefferson, allows a LP not necessary running out of memory to reclaim spaces used for storing positive and negative messages, and also the system states [10]. In this protocol the selection of LP for memory reclamation is more flexible. An artificial rollback scheme that activates the rollback recovery for memory reclamation even without the presence of stragglers, is proposed by Lin [11]. Das and Fujimoto's adaptive memory management scheme (AMM) attempts to automatically adjust Time Warp simulations to obtain optimal performance by controlling the amount of unused memory [2]. In general, such memory management (or optimism control) is able to ensure the proceeding of PDES. However, it is done at the expense of undoing events that have been correctly executed.

\section{The Proposed Two-Sided Adaptive Throttling Scheme}

An ideal throttling mechanism for Time Warp must fulfill three criteria: (i) able to level out the individual LVT progression, (ii) able to maintain sufficient events to keep the CPU utilized, and (iii) does not incur too much overhead. Due to the stochastic behavior of simulation events, the control scheme must also respond dynamically to the proceeding of each LP during program execution. Therefore, it is desirable to use the LVT as an indication of simulation progress in a LP and to throttle its event execution rate, which is the number of events executed per unit CPU time.

\subsection{Global Progress Window}

Since the performance of Time Warp is largely dependent on the even progress of each LP, an indicative measurement of the global simulation progress is crucial to the successful implementation of the throttle. The proposed scheme uses a global progress window (GPW) to indicate the progress status of the slowest and the fastest LPs. Let the local progress time of $L P_{i}$ be denoted as $L P T_{i}$, which is the minimum of the $L V T_{i}$ and the timestamp of each in-transit message sent by $L P_{i}$. Let GFT be the global furthest time. We also represent GPW by [GVT...GFT], where GVT $=\min \left(L P T_{i}\right) \forall i$, and $\mathrm{GFT}=\max \left(L V T_{i}\right) \forall i$. Thus, GPW provides a global time scale for each LP to calibrate its simulation progress. In general, a LP of LVT close to GVT is regarded as slow, and close to GFT as fast. It is noteworthy that GPW can be determined without in- curring a substantial overhead as its acquisition can be embedded in the existing GVT protocols.

\subsection{LVT Progression Control Strategies}

Each LP used in parallel simulation can be implemented by a physical process (PP), such as a PVMI process or a MPI process, etc. As the number of PPs used in PDES is often greater than the number of processors available, each processor will have to execute more than one PP. Consequently, the LVT progression in each LP is directly affected by the active period of the PP implementing it. In the following discussions we explore the feasibility and effectiveness of four strategies used to throttle the LVT progression on a distributed computing environment.

\subsubsection{Changing the Priority of PPs}

By changing the priority of a PP when it is spawned, it is possible to control its probability of being scheduled for execution. Therefore. the PP of a slower LP can be assigned a higher priority and vice versa. The main problem of this scheme is that it is impossible to determine the rate of $\mathrm{LV} T$ progression at compile time. Thus, it is difficult to assign an appropriate priority to the PP representing the LP.

\subsubsection{Changing the CPU Quantum Allocnted to PPs}

The active period of a PP can also be controlled by changing the CPU quantum allocated to it when it is scheduled for execution. This active period in turn controls the LVT progression of the LP during the simulation run. However, this control strategy' becomes ineffective when I/O activities, such as receiving/sending messages from/to communication channels are regularly activated. The PP will be suspended on each I/O request. regardless of the amount of remaining $\mathrm{CPl}^{\circ}$ time. As a result, the LVT progression in the LP cannot be properly controlled.

\subsubsection{Process Migration}

Process migration aims to balance the workload of each processor on the fly by migrating some processes from heavily loaded processors to others with lighter workload. As for PDES the PP of a LP with slower LVT progression can be migrated to a processor with lighter workload. thus increasing its chance of being executed $[15,16]$. However, process migration involves substantial amount of overheads in saving the process context. flushing the communication channels to prevent loss of messages, and broadcasting the new PP location to all 
other PPs. It is noteworthy that such overheads are also linearly proportional to the number of migrations performed. Moreover, process migration may cause an increase in fanout, which is number of communication links to remote LPs, thus adversely increasing the communication overheads.

\subsubsection{Regulating the Period of Event Execu- tion Cycle}

Instead of changing the parameters associated with the PPs or migrating them, this strategy dynamically adjusts the number of events executed in each LP cycle. To begin. figure 1 gives a sketch of the activities in a LP cycle. Each cycle starts with the reception of all messages from the incoming channels. Time Warp protocols, such as GVT computation and message cancellation, will be processed immediately, but event messages will be placed in the input queue. Following the message reception, the first message will be dequeued and executed. A rollback recovery is activated if the event timestamp is less than the LVT.

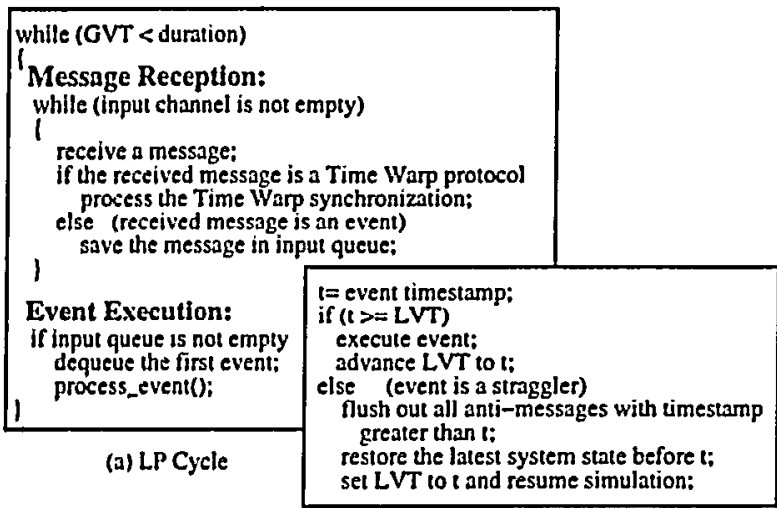

(b) process_event()

Figure 1: Activities Performed in a LP Cycle

The control strategy regulates the period of event execution cycle to achieve an in pace LVT progression. Instead of executing at most one event in each LP cycle, a regulator denoted by $k$, where $k \geq 0$, is used to throttle the number of events for execution. The conventional Time Warp uses $k=1$ throughout the duration of simulation. Intuitively, if LVT progression in a LP is slow. the regulator should be set to a larger value, rather than remaining as a constant, to accelerate the event execution. From the implementation view point, the event regulation approach is more dynamic than the previous three methods. As the regulator value can be adjusted in every cycle, the active period of each PP can alway's be adapted to its local progress. As a result, the event execution rate of the corresponding LP can be fine-tuned based on its LVT progression. This event regulation scheme is used in the proposed throttle.

\subsection{Adaptive Throttle Design}

The runtime behavior of Time Warp simulation can be irregular and highly dependent on the capabilities of the implementation platform, the characteristics of the system to be simulated and the system workload. As such the LPs may exhibit a huge variance of LVTs during the simulation run. To better control the global simulation progress, both the fast and slow LPs must be throttled accordingly. This approach is adopted in the proposed throttle scheme to level out the progression of LVTs.

\subsubsection{Characterizations of Fast and Slow LPs}

For a better classification of LVT status, the GPW is divided into three zones (see figure 2). A hysteresis zone is introduced in the interval to smooth out the small LVT fluctuations. Let $h l$ and $h u$ be the lower and upper bounds of the hysteresis zone respectively. In practice the determination of $h l$ and $h u$ is a complex multi-variate problem. The hysteresis interval can be attributed not only to stochastic behavior of simulation events, but also includes the characteristics of implementation platform, such as communication delays

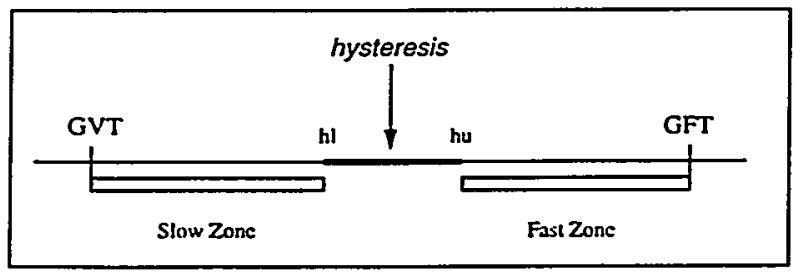

Figure 2: Partitioning Global Progress Window

and system workload. Therefore, a complete search using a set of benchmark programs for the optimal value is time consuming and impractical. After a series of simulation runs, a systematic characterization of the hysteresis zone is adopted. We assume that the hysteresis interval is proportional to that of GPW, i.e., $h u-h l=r \times(G F T-G V T)$, where $0<r \leq 1$. It follows that $h l=(0.5+r / 2) \times G V T+(0.5-r / 2) \times G F T$, and $h u=(0.5-r / 2) \times G V T+(0.5+r / 2) \times G F T$. A LP is classified as slow if its LVT falls within $[G V T . . h l)$, and fast if its LVT falls within (hu...GFT].

\subsubsection{Parameterization of Event Regulator}

It is noteworthy the LVT acceleration done on slow LPs may produce racing effect if it is not managed properly. Consider three LPs positioned on a circuit 
and executed by one processor (see figure $3 \mathrm{a}$ ). Let $L V T_{1}=5, L V T_{2}=10$ and $L V T_{3}=30$, and GPW $=[5$ ...30]. This scenario shows that $L P_{3}$ is a run away process as it receives stragglers from $L P_{1}$ (see figure $3 \mathrm{~b}$ ). Both $L P_{1}$ and $L P_{2}$ are slow as their LVTs are close to GVT. Thus, a larger regulator value can be used in $L P_{1}$ to accelerate the LVT progression. Nonetheless, the acceleration will create causality error in $L P_{1}$ once $L V T_{1} \gg L V T_{2}$ (see figure 3c). Similarly, the same LVT acceleration performed in $L P_{2}$ also creates causality error when $L V T_{2} \gg L V T_{3}$ (see figure 3d). As a result, rollback recovery is activated recursively on the

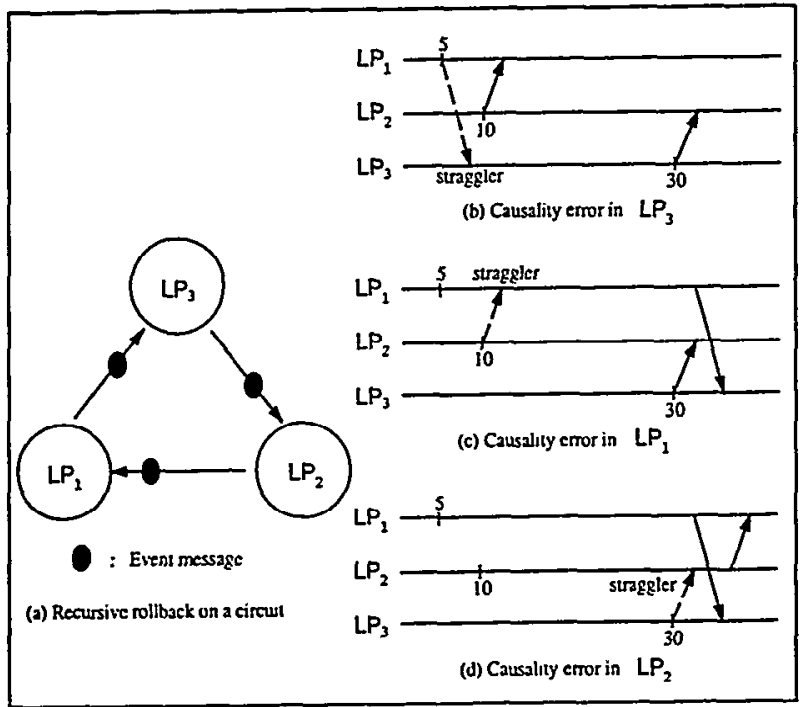

Figure 3: Racing Effect due to Uncontrolled Acceleration

circuit. To prevent the occurrence of such a racing effect, the LVT acceleration done on slow LPs must be constrained. In our throttling scheme the acceleration is terminated when the LVT sweeps pass the upper bound of hysteresis zone. We also bound the regulator by a value $k_{\max }$ so that the LVT acceleration does not exceed the machine capacity.

For a better control of the LVT acceleration, the regulator used in different slow LPs should not only take its LVT status on the GPW into consideration, but also the status of its input buffer. A slow LP should accelerate faster than others if its input buffer contains more pending events, meaning that the timestamp of its last pending event is further away from its LVT. Let $T S($ Last Ev) be the timestamp of the last event message in the input queue. The parameterization of event regulator used is $k=k_{\max } \times \min \left(\frac{T S(L a s t E v)-L V T}{h u-L V T}, 1\right)$, which allows more events to be executed in the LP cycle if the timestamp of the last pending event is further away from the LVT.

\subsubsection{Modification to Activities in LP Cycle}

The added logic used for throttling the LVT progression is summarized in algorithmic form, as shown in figure 4. The values of $h l$ and $h u$ used to bound the hysteresis zone are computed after the values of GVT and GFT have been acquired by the GVT protocol. We henceforth call this the GPW protocol. The duration of a LP cycle used by the throttling scheme is determined by the regulator. As for slow LPs, a larger regulator value is used to accelerate the event execution. For those LPs that fall within the hysteresis zone, the simulation proceeds as normal with at most one event executed in each cycle. As for the fast LP, the regulator is set to 0

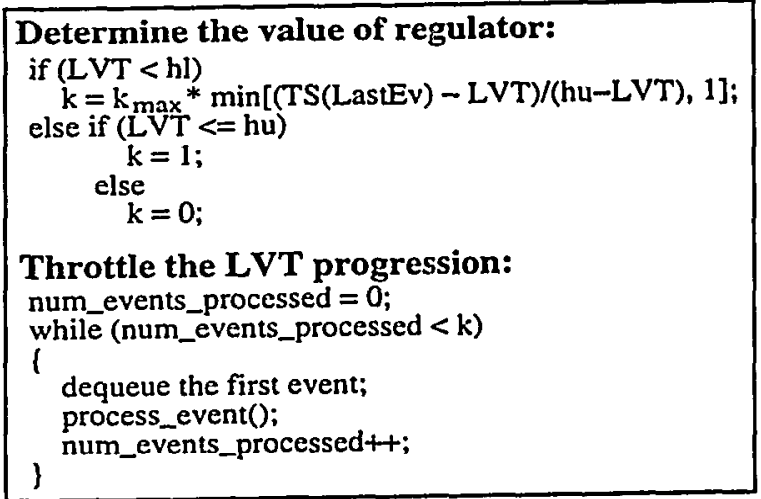

Figure 4: Throttle Logic Used in Event Execution

to prevent it from advancing its LVT further. However, the fast LPs will still have to receive messages from the incoming channels and respond to the synchronization protocols. The GPIV protocol is activated in the first two types of LP when their LV'T passes the hysteresis zone. The objective is to allow fast LPs to resume their event execution after a new GPW is determined.

\section{Performance Analysis}

The Time Warp simulator is currently implemented on a cluster of workstations which mimics a multiprocessor cluster. To handle the spawning, communication, and synchronization of processes, the PVM (Parallel Virtual Machine) library [ 7$]$ is used. The proposed Time Warp throttling mechanism is implemented in a simulation toolkit called SPaDES/C++ [19]. The modular design of SPaDES supports experimental research in synchronization protocols, and ease of parallel simulator development without dealing with the intricacies of simulation synchronization and parallelism.

\subsection{Simulation Applications}

Two examples are used in this study. The first example contains a sequence of service stations arranged 
in a pipeline with exponentially increasing service times (figure 5). This contrived example produces a skewed pattern in LVT progression, thus causing excessive rollback overheads. Our purpose is to illustrate the effectiveness of the proposed throttling scheme in reducing

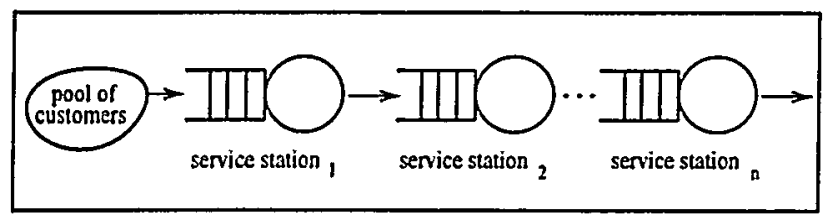

Figure 5: Linear Pipeline Simulation Model $(n=5$ in experimentation)

the number of rollback occurrences. The second example is the timeshared computer system (figure 6) discussed in the book by Hoover and Perry [8]. In both examples exponential distribution is used to model the inter-arrival time and service time, and FIFO queuing discipline is assumed.

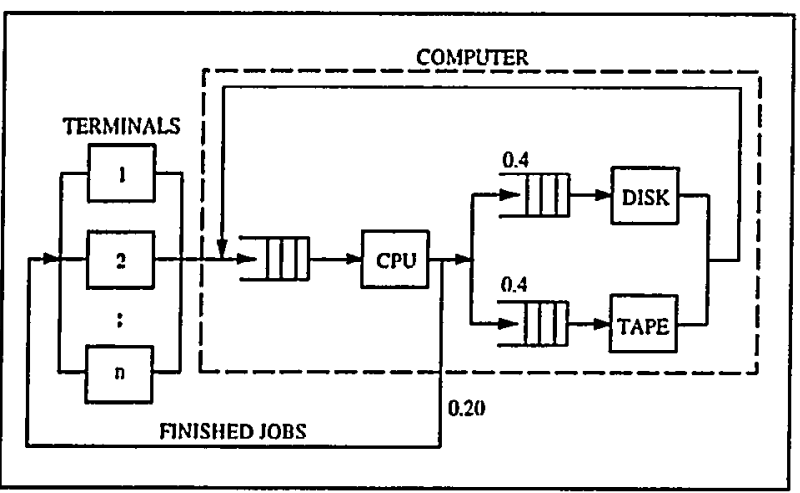

Figure 6: Timeshared Computer Simulation Model ( $n=15$ in experimentation)

\subsection{Experimental Results}

Both the conventional Time Warp and a throttled version, henceforth referred to as ConTW and ThrTW respectively, are implemented in the SPaDES workbench. After performing several set of simulation runs, the following throttle parameters, $r=0.2$, and $k_{\max }=$ 8 , are selected. Due the the multi-user and multitasking operating environment, and different location of workstations used, the communication overhead of each simulation run is high. Work is in progress to port the implementation onto the IBM SP2 and Fujitsu AP3000 parallel computers that have lower communication costs and more predictable communication delays.
Five workstations are used to run the linear pipeline simulator as there are five service stations in the queuing network. As for the timeshared computer simulator, three workstations are used to executed the CPU, disk and tape units. Tables 1 and 2 show that ThrTW is effective in reducing the rollback occurrences in both examples. Moreover, table 1 shows that ConTW ex-

\begin{tabular}{|c|c|c|}
\hline \multirow{2}{*}{$\begin{array}{c}\text { simulation } \\
\text { duration }(\mathrm{sec})\end{array}$} & \multicolumn{2}{|c|}{ number of rollbacks } \\
\hline & ConTW & ThrTW \\
\hline$\overline{50000}$ & $\overline{191}$ & $\overline{150}$ \\
\hline 100000 & 606 & 269 \\
\hline 150000 & 1126 & 358 \\
\hline 200000 & 1654 & 450 \\
\hline 250000 & 2425 & 635 \\
\hline
\end{tabular}

Table 1: Comparison of Rollback Counts (Linear Pipeline)

\begin{tabular}{||c||c|c||}
\hline \hline \multirow{2}{||c||c|}{$\begin{array}{c}\text { simulation } \\
\text { duration (sec) }\end{array}$} & \multicolumn{2}{c||}{ number of rollbacks } \\
\cline { 2 - 3 } & ConTW & ThrTW \\
\hline \hline 200 & 155 & 53 \\
400 & 313 & 168 \\
600 & 471 & 276 \\
800 & 681 & 384 \\
1000 & 838 & 515 \\
1200 & 1014 & 610 \\
1400 & 1190 & 726 \\
\hline \hline
\end{tabular}

Table 2: Comparison of Rollback Counts (Timeshared Computer)

hibits adverse thrashing behavior when the lookahead, which is the service time, along the pipeline is of increasing order. However, such an unfavorable result. can be controlled when ThrTW is used to throttle the $L V T$ progression.

We also recorded the number of events executed and the number of events committed, henceforth referred to as EvEre and EvCom respectively, during the simulation run. Since a rollback can generates more than one anti-message, it is important to study the effect of rollback thrashing. To measure the effect of rollbacks and for comparison across Time Warp implementations. we introduce two new measures called RPE and $c$. RPE or the number of Rollback events Per committed Event is defined as $\frac{E v E x e-E v C o m}{E v C i o m}$. An RPE of value zero is ideal and is equivalent to no rollback occurrence. while a larger RPE reflects a higher cost of the particular Time Warp implementation. This new measure is somewhat similar to CPI (cycles per instruction). which is the metric used to compare different implementations of the same instruction set archi- 
tecture. The relative effectiveness of ThrTW, as compared to ConTW, in reducing the rollback overhead is defined as $\mathcal{E}=1-\frac{R P E_{C_{0 n} T W}-R P E_{T h R T W}}{R P E_{C_{0}} T W}$. An $\mathcal{E}$ of value one indicates no improvement in the effectiveness, greater than one indicates deteriorated effectiveness (more costly), less than one indicates improved effectiveness (less costly), and zero indicates the best effectiveness (no rollback).

Tables 3 and 4 shows that the proposed throttling scheme reduces the rollback cost of Time Warp simulation significantly. As for different durations of simulation run, ThrTW is able to maintain a fairly stable cost as compared to that of ConTW. The pipeline example with exponential growth of service time (refer to table 3) shows a stable cost for ThrTW when simulation duration is longer while ConTW shows an increasing cost as the duration is increased. The $\mathcal{E}$ indicator in both examples shows that ThrTW is effective in reducing the rollback cost as compared to ConTW. In particular, table 3 shows that as the simulation duration is increased in the pipeline example so is the effectiveness of ThrTW in reducing the rollback cost. A significant reduction in RPE is also observed from the averaged $R P E_{C o n T w}$ of 8.07 to the averaged $R P E_{T h r T W}$ of 1.21 in the timeshared computer example (refer to table 4 ).

Figures 7 and 8 show the elapsed time of ThrTW is better than ConTW in both examples. This effect is attributed to the reduction in rollback thrashing when the throttling scheme is used. As a result, the time incurred in restoring the system states, undoing the side effects and re-executing the simulation events is decreased, giving a smaller program elapsed time.

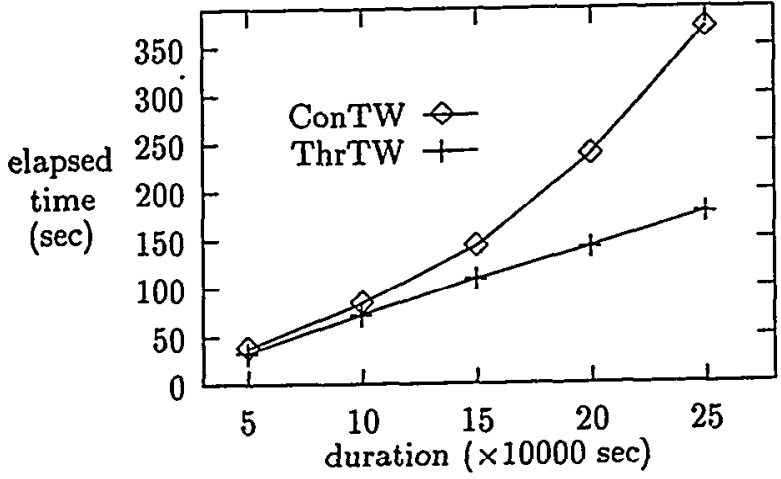

Figure 7: Elapsed Time for Linear Pipeline Simulation

\section{Conclusions}

We have developed a two-sided throttling scheme to reduce the rollback overheads in Time Warp simulation. A new concept called global progress window is used for calibrating the simulation progress in each LP.

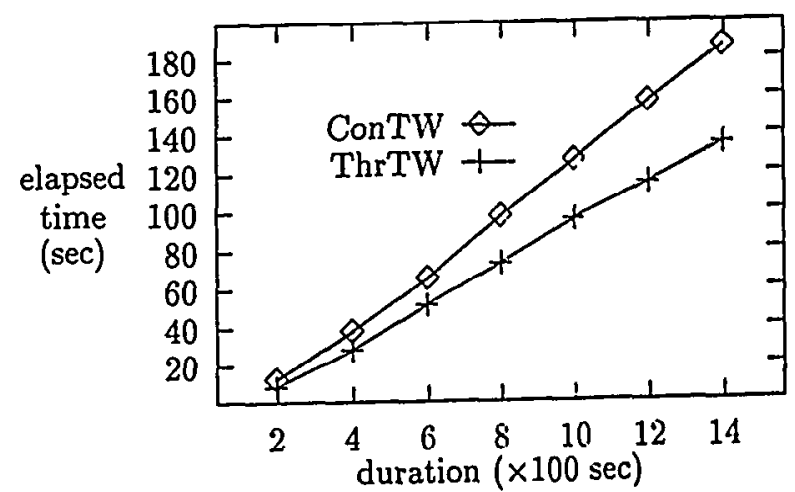

Figure 8: Elapsed Time for Timeshared Computer Simulation

The proposed throttle accelerates the slower LPs based on their simulator progress and the input queue status, and suspends the LVT progression in faster LPs to lessen the potential of causality crror. As no additional global synchronization is used in the proposed throttle, the overhead incurred is insignificant. We quantify the rollback effect by proposing the use of two new measures called RPE (number of Rollback events Per committed Events), and $\mathcal{E}$ (relative Effectiveness in reducing rollback overhead). These are also important measures especially for comparing different Time Warp implementations. In the timeshared computer system simulation example, the proposed throttle reduces the RPE from 8.07 to 1.21 on the average, and the total number of events executed by more than $70 \%$. Our experimental result reveals that the proposed throttle is effective in regulating the proceeding of each simulation process, resulting in a significant reduction of rollback occurrences and improvement of simulation runtime. An added advantage of the throttle is its consistent performance for different simulation durations and lookaheads. Such a merit is not found in the conventional Time Warp simulation.

\section{References}

[1] D. Ball and S. Hoyt, "The Adaptive Time-Warp Concurrency Control Algorithm," Proc. of the SCS Multiconference on Distributed Simulation, pp. 174-177, 1990.

[2] S. R. Das and R. M. Fujimoto, "An Adaptive Memory Management Protocol for Time II arp Parallel Simulation," ACM SIGMETRICS Conference on Measurement and Modeling of Computer Systems, pp. 201-210, 1994.

[3] S. R. Das, "Estimating the Cost of Throttled Execution in Time Warp," Proc. of 10th Workshop on Parallel and Distributed Simulation, pp. 186-189, 1996. 


\begin{tabular}{|c|c|c|c|c|c|c|}
\hline \multirow{2}{*}{$\begin{array}{c}\text { simulation } \\
\text { duration }(\mathrm{sec})\end{array}$} & \multirow[b]{2}{*}{ EvCom } & \multicolumn{2}{|c|}{ ConTW } & \multicolumn{2}{|c|}{ ThrTW } & \multirow[b]{2}{*}{$\mathcal{E}$} \\
\hline & & EvExe & $R P E_{\text {ConTW }}$ & EvExe & $R P E_{T h r T W}$ & \\
\hline 50000 & $\overline{4125}$ & 6405 & $\overline{0.55}$ & $\overline{6077}$ & $\overline{0.47}$ & $\overline{0.86}$ \\
\hline 100000 & 8241 & 18282 & 1.22 & 12373 & 0.50 & 0.41 \\
\hline 150000 & 12372 & 30767 & 1.49 & 18394 & 0.49 & 0.33 \\
\hline 200000 & 16449 & 51633 & 2.14 & 24289 & 0.48 & 0.22 \\
\hline 250000 & 20659 & 72197 & 2.49 & 31206 & 0.51 & 0.20 \\
\hline
\end{tabular}

Table 3: Comparison of Time Warp Implementations (Linear Pipeline)

\begin{tabular}{||c||c||c|c||c|c||c||}
\hline \hline \multirow{2}{*}{$\begin{array}{c}\text { simulation } \\
\text { duration (sec) }\end{array}$} & \multirow{2}{*}{ EvCom } & \multicolumn{2}{c||}{ ConTW } & \multicolumn{2}{c||}{ ThrTW } & \multirow{2}{*}{} \\
\cline { 3 - 7 } & EvExe & $R P E_{\text {ConTW }}$ & EvExe & $R P E_{\text {ThrTW }}$ & $\mathcal{E}$ \\
\hline \hline 200 & 193 & 1613 & 7.37 & 426 & 1.21 & 0.16 \\
400 & 395 & 3443 & 7.72 & 853 & 1.16 & 0.15 \\
600 & 618 & 5432 & 7.79 & 1384 & 1.24 & 0.16 \\
800 & 851 & 7779 & 8.14 & 1901 & 1.23 & 0.15 \\
1000 & 1091 & 10075 & 8.23 & 2423 & 1.22 & 0.15 \\
1200 & 1356 & 12708 & 8.37 & 2988 & 1.20 & 0.14 \\
1400 & 1538 & 15241 & 8.91 & 3366 & 1.19 & 0.13 \\
\hline \hline
\end{tabular}

Table 4: Comparison of Time Warp Implementations (Timeshared Computer)

[4] A. Ferscha, "Parallel and Distributed Simulation of Discrete Event Systems," in Handbook of Parallel and Distributed Computing, McGraw-Hill, 1995.

[5] A. Ferscha and J. Luethi, "Estimating Rollback Overhead for Optimism Control in Time Warp," Proc. of the 28th Annual Simulation, pages 2-12, 1995.

[6] A. Gafni, "Rollback Mechanisms for Optimistic Distributed Simulation Systems," Proc. of the SCS Multiconference on Distributed Simulation, Vol. 19(3), pp. 61-67, 1988.

[7] A. Geist, A. Beguelin, J. Dongarra, W. Jiang, R. Manchek and V. Sunderam, "PVM: Parallel Virtual Machine - A User's Guide and Tutorial for Networked Parallel Computing," The MIT Press, 1994.

[8] S. V. Hoover and R. F. Perry, "Simulation-A ProblemSolving Approach", Addison-Wesley Publishing Company, 1989 .

[9] D. R. Jefferson, "Virtual Time," ACM Transactions on Programming Languages and Systems, Vol. 7(3), pp. 404-425, July 1985.

[10] D. R. Jefferson, "Virtual Time II: Storage Management in Distributed Simulation," Proc. of the 9th Annual ACM Symposium on Principles of Distributed Computing, pp. 75-89, 1990.

[11] Y. B. Lin and B. R. Preiss, "Optimal Memory Management for Time Warp Parallel Simulation," ACM Transactions on Modelling and Computer Simulation, Vol, 1(4), pp. 283-307, October 1991.

[12] V. K. Madisetti and D. A. Hardaker, "The MIMIDIX Environment for Parallel Simulation," Journal of Par- allel Distributed Computing, Vol. 18, pp. 473-483, 1993.

[13] J. Misra, "Distributed Discrete-Event Simulation," Computing Surveys, Vol. 18(1), pp. 39-65, March 1986.

[14] J. Misra and K. M. Chandy, "Asynchronous Distributed Simulation via a Seguence of Parallel Computations," Communication of the ACM, Vol. 24(4), pp. 198-206, April 1981.

[15] Peter L. Reiher and David Jefferson, "Virtual Time Based Dynamic Load Management in the Time Warp Operating System," Proc. of the ACM/IEEE/SCS Workshop on Parallel and Distributed Simulation (PADS), pp. 103-111, 1990.

[16] Rolf Schlagenhaft, Martin Ruhwandl, Christian Sporrer and Herbert Bauer "Dynamic Load Balancing of a Multi-Cluster Simulator on a Network of Workstations," Proc. of the ACM/IEEE/SCS Workshop on Parallel and Distributed Simulation (PADS), pp. 175180, 1995.

[17] J. S. Steinman, “SPEEDES: A Multiple Synchronization Environment for Parallel Discrete-Event Simulation, "International Journal in Computer Simulation, Vol. 2, pp. 251-286, 1992.

[18] J. S. Steinman, "Breathing Time Warp," Proc. of the 7 th Workshop on Parallel and Distributed Simulation, pp. 109-118, 1993.

[19] Y. M. Teo, S. C. Tay and S. T. Kong, "Structured Parallel Simulation of Discrete-Event Systems," Proc. of Sixth Parallel Computing Workshop, Kawasaki, JAPAN, pp. 2-A-1 to 2-A-10, Nov. 12-13, 1996. 